\title{
Analysis of the Real-Time Oncology Review (RTOR) Pilot Program for Approvals of New Molecular Entities
}

\author{
Catherine Feng, PharmD ${ }^{1} \cdot$ Riddhi Virparia, PharmD, RAC ${ }^{2}$ - Eric T.-K. Mui, PharmD, RAC ${ }^{2}$
}

Received: 20 January 2021 / Accepted: 9 April 2021 / Published online: 28 April 2021

(c) The Drug Information Association, Inc 2021

\begin{abstract}
The United States Food and Drug Administration (FDA) Oncology Center of Excellence (OCE) established the Real-Time Oncology Review (RTOR) pilot program in 2017 to streamline the review process for oncology drug applications with the applicant and the Agency agreeing upon a piecemeal strategy and timeline for module components. The Prescription Drug User Fee Act (PDUFA) review clock does not officially start until the final component is submitted. Participation requires careful planning of time and resources due to the multiple submissions and interactions with the FDA. Applicants must also meet certain criteria regarding the clinical trial design and development program to be eligible for RTOR. Publicly available databases (Drugs@FDA) and documents were searched for all RTOR applications, which revealed a total of 28 approved applications that participated from February 2018 to August 2020. Initial marketing applications were further reviewed to identify any potential advantages or limitations from participation in the pilot program. These four case studies demonstrated an individualized RTOR process reflecting the program's pilot status. The FDA approved 3 out of the 4 applications approximately three to four months before the PDUFA goal date. The time savings is not guaranteed as other parts of the review may influence the overall timeline. However, the optional biweekly teleconferences increased communication and collaboration between the applicant and the FDA. The full impact of RTOR on applications remains undetermined as the number of approved applications that have participated in the pilot program is still relatively small.
\end{abstract}

Keywords US Food and Drug Administration · Real-Time Oncology Review · Oncology Center of Excellence · Oncology · Drug Approvals

\section{Introduction}

The United States Food and Drug Administration (FDA) Oncology Center of Excellence (OCE) was first established in 2017 with the goal of achieving patient-centered regulatory decision-making through innovation and collaboration [1]. As one of several initiatives led by OCE, the RealTime Oncology Review (RTOR) program was initially

Catherine Feng, Riddhi Virparia, Eric T.-K. Mui-Co-primary authors

Catherine Feng

cathf2012@gmail.com

1 Pharmaceutical Industry Fellowship Program, Northeastern University, 360 Huntington Ave, R218 140 The Fenway, Boston, MA 02115, USA

2 Division of Pharmacotherapy \& Experimental Therapeutics, UNC Eshelman School of Pharmacy, Chapel Hill, NC, USA piloted in February 2018 to streamline and improve efficiency of the review process for supplemental applications in oncology. In December 2018, the program was expanded to include review of original drug applications [2].

Participation in RTOR does not guarantee approval for an application nor is it intended to enable early action prior to the Prescription Drug User Fee Act (PDUFA) date [3]. Through RTOR, the applicant and the FDA agree upon a piecemeal strategy and timeline for submission components and supportive datasets, which allows the Agency to begin reviewing key efficacy and safety data prior to what is considered the "complete" dossier submission for a New Drug Application (NDA) or Biologics License Application (BLA) [4]. This provides the Agency an earlier opportunity to ask questions and provide feedback about any important regulatory issues that should be addressed [3]. It is important to distinguish from Rolling Review in which complete modules are submitted prior to the full application [2]. In addition, 
the PDUFA review clock does not officially start until the applicant submits the final component.

To meet the eligibility criteria for participation in RTOR, the drug should demonstrate substantial improvements over available therapy (e.g., drugs that were previously granted Breakthrough Therapy Designation [BTD] for the same or other indications, drugs eligible for other expedited pathways) and be evaluated through studies with straightforward designs and easily interpretable endpoints (e.g., overall survival [OS], progression-free survival [PFS]). More complex submissions may also be eligible for RTOR, but the decision is up to the FDA's discretion [3].

Frequent communication usually occurs between the applicant and the Agency during the ongoing submission of all the components. Figure 1 illustrates the general timeline for RTOR proposed by OCE, although it is likely to vary for each application; for comparison, key lifecycle milestones for Piqray (alpelisib) and Qinlock (ripretinib) are also noted. Typically, the applicant first submits topline efficacy and safety data to the FDA and discusses participation via teleconference. If both parties deem that RTOR is appropriate, the applicant starts sending pre-submission data and documents to the Agency based on the agreed upon timelines, which should follow approximately 2 to 4 weeks after the patient database has been locked. In theory, by the time the full application is submitted, the review team is familiar enough with the application to enable a much more efficient review [3].

This article analyzes the four full applications that were approved through RTOR from February 2018 to August 2020 to identify any potential advantages or limitations from participation in the pilot program.

\section{Methods}

We obtained a partial list of oncology applications participating in the RTOR pilot program from previous research which provided participants from program inception in February 2018 to April 2020 [2].This initial list was expanded to include RTOR participants between April 2020 and August 2020 by manual review of the FDA's announcements regarding oncology drug approvals [5]. Applications that participated in the RTOR pilot program but did not ultimately obtain approval were not included, and participation in other pilot programs (Assessment Aid [AAid] and Project Orbis) or other expedited review programs was incidental and not required as part of participation in RTOR. This combined list was further separated into two groups: initial marketing applications supporting a new molecular entity (NDAs or

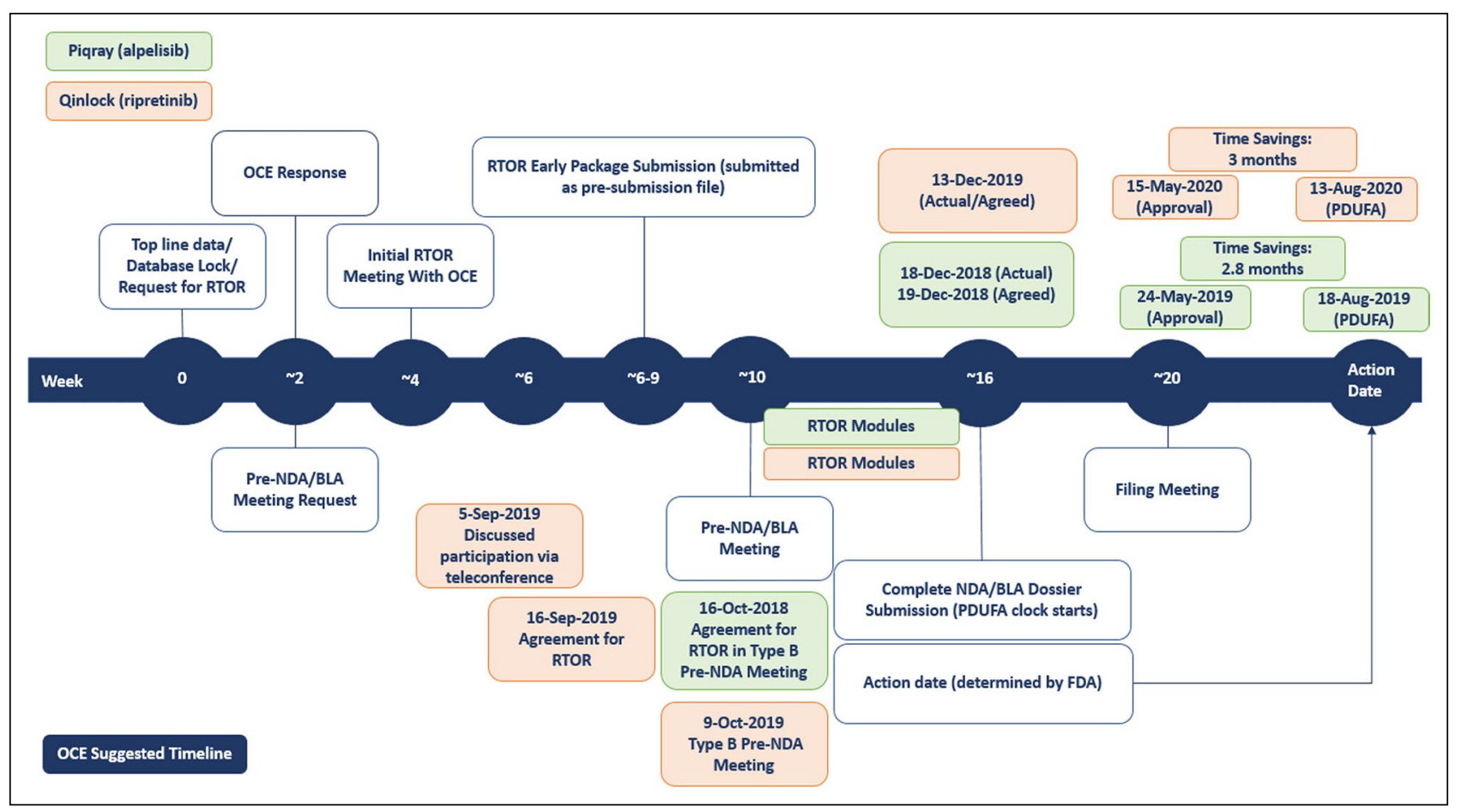

Fig. 1 Illustration of the timelines for Piqray (alpelisib) and Qinlock (ripretinib) in comparison to the Oncology Center of Excellence (OCE) suggested timeline. *Adapted from OCE Suggested Timeline [3]. RTOR Real-Time Oncology Review Program, FDA United
States Food and Drug Administration, OCE Oncology Center of Excellence, NDA New Drug Application, BLA Biologics License Application, PDUFA Prescription Drug User Fee Act 
BLAs) and supplements to an initial marketing application. For each of the initial marketing applications, we performed a retrospective review of the publicly available documents (approval letters, labeling, application review files, and meeting transcripts) on the Drugs@FDA database.

No Institutional Review Board or ethics review was obtained for this study since human subjects were not involved.

\section{Results}

In this analysis, our search revealed a total of 28 approved applications that participated in the RTOR pilot program from February 2018 to August 2020 (see Appendix S1). These applications encompassed a total of 23 different products including 13 small molecules (SM), 7 monoclonal antibodies (mAb), and 3 antibody-drug conjugates (ADC). RTOR applications spanned a wide range of oncologic indications with the highest number for breast cancer (4), chronic lymphocytic leukemia/small lymphocytic lymphoma (4), multiple myeloma (3), and non-small cell lung cancer (3). Supplements to initial marketing applications were mainly filed to support new indications (21) with a smaller proportion representing labeling changes (2) and new dosing regimens (1).

Initial marketing applications represented 4 of the RTOR applications (see Table 1), including 3 small molecules and 1 ADC. Due to publicly available information, these 4 marketing applications were selected out of the 28 and are further described in the case studies below. An advisory committee meeting was held for 1 of these 4 initial marketing applications. These marketing applications received Priority Review (4/4), BTD (3/4), Fast Track (2/4), and integrated other review programs such as Project Orbis (2/4), AAid (4/4), and Chemistry, Manufacturing, and Controls (CMC) AAid (2/4). Out of the 4 marketing applications, one application was granted accelerated approval. The median time between the first RTOR submission to the complete application submission could not be calculated due to the limited publicly available information. The median time from the clinical trial data cut-off to the complete application submission was 5.85 months. The pivotal clinical trials supporting approvals are outlined in Table 2.

\section{Case 1: Piqray (alpelisib)}

Piqray (alpelisib) is an oral inhibitor of phosphatidylinositol-3-kinase (PI3K) with inhibitory activity predominantly

Table 1 Full New Drug Application (NDA) and Biologics License Application (BLA) Real-Time Oncology Reviews

\begin{tabular}{|c|c|c|c|c|c|c|c|c|}
\hline Brand (generic) & Application & Submission date & Approval date & PDUFA date & $\begin{array}{l}\text { Time } \\
\text { savings } \\
\text { (months) }\end{array}$ & $\begin{array}{l}\text { Expedited/review } \\
\text { pathway utilized }\end{array}$ & $\begin{array}{l}\text { Referral to } \\
\text { advisory com- } \\
\text { mittee }\end{array}$ & Orphan \\
\hline Piqray (alpelisib) & NDA 212526 & 18-Dec-18 & 24-May-19 & 18-Aug-19 & 2.8 & $\begin{array}{l}\text { RTOR } \\
\text { AAid } \\
\text { Priority review }\end{array}$ & No & No \\
\hline Tukysa (tucatinib) & NDA 213411 & 20-Dec-19 & 17-Apr-20 & 20-Aug-20 & 4.1 & $\begin{array}{l}\text { Fast Track } \\
\text { BTD } \\
\text { Project Orbis } \\
\text { RTOR } \\
\text { AAid (CMC } \\
\text { AAid) } \\
\text { Priority review }\end{array}$ & No & Yes \\
\hline $\begin{array}{l}\text { Qinlock } \\
\text { (ripretinib) }\end{array}$ & NDA 213973 & 13-Dec-19 & 15-May-20 & 13-Aug-20 & 3.0 & $\begin{array}{l}\text { Fast Track } \\
\text { BTD } \\
\text { Project Orbis } \\
\text { RTOR } \\
\text { AAid (CMC } \\
\text { AAid) } \\
\text { Priority review }\end{array}$ & No & Yes \\
\hline $\begin{array}{l}\text { Blenrep (belan- } \\
\text { tamab mafodo- } \\
\text { tin-blmf) }\end{array}$ & BLA 761158 & 5-Dec-19 & 5-Aug-20 & 5-Aug-20 & 0 & $\begin{array}{l}\text { Accelerated } \\
\text { Approval } \\
\text { BTD } \\
\text { RTOR } \\
\text { AAid } \\
\text { Priority review }\end{array}$ & Yes & Yes \\
\hline
\end{tabular}

AAid Assessment Aid, BLA Biologics License Application, BTD Breakthrough Therapy Designation, CMC Chemistry, Manufacturing, and Controls, NDA New Drug Application, PDUFA Prescription Drug User Fee Act, RTOR Real-Time Oncology Review Program 
Table 2 Overview of pivotal trials to support labeling claims for initial marketing applications

\begin{tabular}{|c|c|c|c|c|c|}
\hline Brand (generic) & Trial & Phase & Study design & $\begin{array}{l}\text { Primary } \\
\text { endpoint }\end{array}$ & $\begin{array}{l}\text { Time from data cut-off } \\
\text { to complete submission } \\
\text { (months) }\end{array}$ \\
\hline Piqray (alpelisib)[17] & $\begin{array}{l}\text { SOLAR-1 (NCT02437318) } \\
\text { Data Cut-Off = } \\
\text { 12-June-18 }\end{array}$ & Phase 3 & $\begin{array}{l}\text { Randomized (1:1) } \\
\text { Double-blind } \\
\text { Placebo-controlled } \\
\text { Multicenter trial } \\
\mathrm{N}=572\end{array}$ & PFS & 6.2 \\
\hline Tukysa (tucatinib) [17] & $\begin{array}{l}\text { HER2CLIMB (NCT02614794) } \\
\text { Data Cut-Off = } \\
\text { 4-Sep-19 }\end{array}$ & Phase 2 & $\begin{array}{l}\text { Randomized }(2: 1) \\
\text { Double-blind } \\
\text { Placebo-controlled } \\
\text { Multicenter trial } \\
\mathrm{N}=612\end{array}$ & PFS & 3.5 \\
\hline Qinlock (ripretinib) [24] & $\begin{array}{l}\text { INVICTUS } \\
\text { (NCT03353753) } \\
\text { Data Cut-Off = } \\
\text { 31-May-19 }\end{array}$ & Phase 3 & $\begin{array}{l}\text { Randomized }(2: 1) \\
\text { Double-blind } \\
\text { Placebo-controlled } \\
\text { Multicenter trial } \\
\mathrm{N}=129\end{array}$ & PFS & 6.4 \\
\hline $\begin{array}{l}\text { Blenrep (belantamab mafodo- } \\
\text { tin-blmf) [30] }\end{array}$ & $\begin{array}{l}\text { DREAMM-2 } \\
\text { (NCT03525678) } \\
\text { Data Cut-Off = } \\
\text { 21-June-19 }\end{array}$ & Phase 2 & $\begin{array}{l}\text { Open-label } \\
\text { Randomized (1:1) } \\
\text { Single-arm (two dose cohorts) } \\
\text { Multicenter trial } \\
\mathrm{N}=221\end{array}$ & ORR & 5.5 \\
\hline
\end{tabular}

ORR Overall Response Rate, PFS Progression-Free Survival

against PI3K $\alpha$ [6]. Piqray, manufactured by Novartis Pharmaceuticals Corporation (the Sponsor), was reviewed by the Division of Oncology Products (DOP1) and was approved on May 24, 2019 for the treatment of postmenopausal women and men, with hormone receptor-positive, human epidermal growth factor receptor 2 (HER2)-negative, PIK3CA-mutated, advanced or metastatic breast cancer as detected by an FDA-approved test following progression or after an endocrine-based regimen [7, 8]. Piqray was the first NDA for a new molecular entity to gain approval under the RTOR pilot program [9]. In addition, the application was granted Priority Review and utilized the AAid, resulting in an approval approximately 3 months ahead of the PDUFA VI deadline of August 18, 2019. Piqray was not referred to an FDA advisory committee because the application did not raise significant safety or efficacy issues.

The agreement to participate in the RTOR pilot program and the AAid occurred during a Type B pre-NDA meeting between the Sponsor and the FDA to discuss submission of an NDA based on the results of the pivotal clinical study, SOLAR-1 (NCT02437318) [10]. As part of this program, both parties agreed on the content for a complete application as well as timelines to supply each module component. The final agreed upon modules and dates (Appendix S2) resulted in a piecemeal submission strategy spanning approximately 2 months with a final NDA dossier proposed for December 19, 2018. The first submission components consisted of Module 3 manufacturer information, followed closely by nonclinical study reports, some clinical datasets, and package insert information. Module 2 summary documents, the AAid, and the complete Quality (Module 3) dossier were planned for submission approximately one month into the RTOR submission process. Final components included supplementary summary documents and supporting clinical documentation.

The FDA also agreed to standing bi-weekly teleconferences with the Sponsor with the option for additional standing teleconferences to discuss CMC components of the file. The FDA also stated that the Sponsor would have to submit the updated documents with tracked changes, summary of changes and the final version. Other key FDA meetings for Piqray included a Type B End-of-Phase 2 meeting with the FDA as part of clinical development to discuss the design of the pivotal clinical trial, SOLAR-1 [6].

SOLAR-1 was a Phase 3, randomized, double-blind, placebo-controlled study of alpelisib in combination with fulvestrant for men and postmenopausal women with hormone receptor-positive, HER2-negative advanced breast cancer which progressed on or after aromatase inhibitor treatment. The primary endpoint was PFS between populations dosed with alpelisib and fulvestrant versus those dosed with placebo and fulvestrant. In addition, treatment effect was evaluated in two sub-populations consisting of patients with PIK3CA mutated tumors and those without. In the sub-population with PIK3CA mutations, PFS at a median follow-up of 20 months was 11.0 months (95\% confidence interval [CI] 7.5 to 14.5$)$ in the alpelisib group versus 5.7 months (95\% CI 3.7 to 7.4 ) in the placebo group (hazard ratio [HR] 0.65 , 
95\% CI 0.5 to $0.85, \mathrm{P}<0.001)$. In those without PIK3CA mutations, the HR was 0.85 (95\% CI 0.58 to 1.25$)$ [11, 12].

\section{Case 2: Tukysa (tucatinib)}

Tukysa (tucatinib) is an oral tyrosine kinase inhibitor of HER2. Tukysa, developed by Array BioPharma, Inc. and licensed to Cascadian Therapeutics, Inc. (acquired by Seattle Genetics, Inc.), was reviewed by Division of Oncology 1 (DO1) and approved on April 17, 2020 for the use in combination with trastuzumab and capecitabine for treatment of adult patients with advanced unresectable or metastatic HER2-positive breast cancer, including patients with brain metastases, who have received one or more prior antiHER2-based regimens in the metastatic setting [13-15]. In addition to being a part of the RTOR pilot, this application was reviewed as a part of Project Orbis, in which the FDA collaborated with Australian Therapeutic Goods Administration (TGA), Health Canada, Health Sciences Authority (HSA, Singapore) and Swissmedic (SMC, Switzerland) [14]. Furthermore, this application was granted Priority Review, BTD, Fast Track, and Orphan Drug Designation (ODD) and participated in the AAid as well as the CMC AAid [13, 14]. This resulted in an approval approximately 4 months ahead of the PDUFA deadline (August 20,2020) [13]. Tukysa was not referred to the advisory committee because it did not raise significant safety or efficacy issues that were unexpected for a drug of this class or in the intended population, and there were no controversial issues for discussion [16].

The Sponsor agreed to participate in both the RTOR pilot and Project Orbis on October 21, 2019. To note, this agreement occurred after the Type B pre-NDA meeting on February 25, 2019 in which the content and format of the planned NDA was discussed. The timelines for RTOR and content for submission batches are not publicly available. Other key FDA interactions for Tukysa included a Type B End-of-Phase 1 meeting (October 11, 2016) and receipt of preliminary comments with the Agency's acceptance of the modifications to the HER2CLIMB protocol (October 24, 2018; canceled the planned Type B meeting) [13].

Approval for Tukysa was based on the efficacy and safety results of HER2CLIMB, a randomized (2:1), double-blind, placebo-controlled, Phase 2 clinical trial. The trial enrolled 612 patients who had HER2-positive metastatic breast cancer with or without brain metastases previously treated with trastuzumab, pertuzumab, and trastuzumab emtansine to receive either tucatinib or placebo, in combination with trastuzumab and capecitabine. The overall population consisted of approximately $48 \%$ of patients who had brain metastases or a history of brain metastases at the time of study entry. The primary endpoint was PFS, assessed in the first 480 patients who were randomized. At one year, PFS in the tucatinib-combination was $33.1 \%$ and $12.3 \%$ in the placebo-combination group (HR for disease progression or death, $0.54 ; 95 \%$ CI 0.42 to $0.71 ; \mathrm{P}<0.001)$. The median duration of PFS was 7.8 months in the tucatinibcombination patients compared to 5.6 months in the placebo-combination group. Secondary endpoints included OS, PFS among patients with brain metastases at baseline, confirmed objective response rate, and safety in the total population (612 patients). In the tucatinib-combination group and the placebo-combination group, the OS at 2 years was $44.9 \%$ and $26.6 \%$ (HR for death, 0.66 ; 95\% CI 0.50 to $0.88 ; \mathrm{P}=0.005$ ), and the median OS was 21.9 months and 17.4 months, respectively. In both of these groups, PFS at 1 year among the patients with brain metastases was $24.9 \%$ in the tucatinib-combination group and $0 \%$ in the placebo-combination group (HR, $0.48 ; 95 \%$ CI 0.34 to $0.69 ; \mathrm{P}<0.001)$, and the median PFS was 7.6 months and 5.4 months, respectively [18].

\section{Case 3: Qinlock (ripretinib)}

Qinlock (ripretinib) is a tyrosine kinase inhibitor (TKI) that broadly inhibits KIT proto-oncogene receptor tyrosine kinase (KIT) and platelet-derived growth factor receptor A (PDGFRA) kinase signaling, including wild type, primary, and secondary mutations [19]. Qinlock, manufactured by Deciphera Pharmaceuticals, was reviewed by Division of Oncology 3 (DO3) and approved on May 15, 2020 for the treatment of adult patients with advanced gastrointestinal stromal tumor (GIST) who have received prior treatment with 3 or more kinase inhibitors, including imatinib [20].The approval came three months earlier than the goal PDUFA date of August 13, 2020 [21]. Qinlock had previously been granted BTD, Fast Track, and ODD in addition to Priority Review [22]. Referral to an advisory committee was deemed unnecessary because Qinlock was not first in class and there were no significant safety or efficacy issues seen in the intended population [20].

In an informal teleconference held on September 5, 2019, Deciphera discussed their plans with the Agency for potential participation in each of the FDA's OCE pilot programs including RTOR, Project Orbis, and the AAid (including CMC AAid) [21]. Shortly thereafter, on September 16, 2019, Deciphera decided to participate in all three programs, less than one month before the Type B pre-NDA meeting on October 9, 2019. As part of RTOR, both parties came to an agreement on the rolling submission plan, including timelines for each module and its components. This piecemeal strategy included 6 submission batches spanning less than 2 months with the final NDA dossier proposed for December 13, 2019. Only some Module 3 information was submitted in the initial batch (manufacturing information) with remaining components submitted last. Module 5 components were spread out over most of the batches while the submission 
of Module 2 and Module 4 components began from the third batch. Other key FDA meetings for Qinlock included an End-of-Phase 1/Pre-Phase 3 meeting to discuss whether the nonclinical and clinical pharmacology plans and study design of the Phase 3 trial were adequate to support an NDA filing. An End-of-Phase 2 meeting regarding product quality was also discussed as part of the clinical development program to support registration plans [23].

Approval for Qinlock was based on the pivotal Phase 3 INVICTUS trial, which demonstrated that ripretinib prolonged PFS compared to placebo in patients with advanced GIST whose disease had progressed on or who were intolerant to imatinib, sunitinib, and regorafenib. In this randomized, double-blind, placebo-controlled, multicenter trial, 129 patients were randomized 2:1 to receive either ripretinib $150 \mathrm{mg}$ or placebo once daily. The primary efficacy endpoint was PFS and the secondary efficacy endpoint was objective response rate (complete or partial response as assessed by blinded independent central review). Overall, ripretinib prolonged median PFS as assessed by blinded independent central review (BICR) by 6.3 months (95\% CI 4.6-6.9 months) vs. 1.0 month (95\% CI 0.9-1.7 months) with placebo (HR 0.15 ; 95\% CI $0.09-0.25 ; \mathrm{P}<0.0001)$ with an objective response rate of $9.4 \%(\mathrm{P}=0.0504)$. Key inclusion criteria included patients 18 years or older diagnosed with GIST with at least one measurable lesion according to modified Response Evaluation Criteria in Solid Tumors version 1.1 (mRECIST 1.1). Median OS was 15.1 months (95\% CI 12.3-15.1 months) with ripretinib compared to 6.6 months (95\% CI 4.1-11.6 months) in the placebo group (HR 0.36; 95\% CI 0.21-0.62). However, statistical significance for OS could not be determined since hierarchical testing was used on the endpoints, and objective response rate was also not significant [24].

\section{Case 4: Blenrep (belantamab mafodotin)}

Blenrep (belantamab mafodotin-blmf) is a B-cell maturation antigen (BCMA)-directed antibody and microtubule inhibitor conjugate. Blenrep, manufactured by GlaxoSmithKline, was granted accelerated approval on August 5, 2020 for the treatment of adult patients with relapsed or refractory multiple myeloma (RRMM) who have received at least 4 prior therapies, including an anti-CD38 $\mathrm{mAb}$, a proteasome inhibitor (PI), and an immunomodulatory agent [25]. The review used RTOR in addition to the AAid, and the application was granted ODD, BTD, and Priority Review [26]. Blenrep was reviewed by the Division of Hematologic Malignancies II (DHM2) and approval was achieved on the PDUFA goal date (August 5, 2020) [27]. This application was discussed at the Oncology Drug Advisory Committee (ODAC) meeting on July 14,2020 . The discussions held were based on the benefit-risk profile for this medication in the proposed population in the context of ocular toxicity $[27,28]$.

With regards to the contents of the BLA, the clinical contents were discussed in a Type B pre-BLA meeting held in May 2019, and an additional Type B CMC pre-BLA meeting was held in July 2019. The Sponsor had agreed to participate in the RTOR program after the pre-BLA meetings. They agreed to submit the RTOR batches through November 2019 with the final batch in December 2019. The timelines and content for RTOR submission batches are not publicly available. Approval of the BLA was achieved on the PDUFA date of August 5, 2020, subsequent to the July 14, 2020 ODAC meeting $[28,29]$.

Accelerated approval was based on data from the pivotal DREAMM-2 study, a Phase 2, open-label, single-arm, multicenter study in patients with RRMM who had received at least 3 prior lines of therapy including a PI, an immunomodulatory agent and an anti-CD38 mAb. The primary efficacy endpoint of overall response rate (ORR), assessed by an Independent Review Committee, was defined as the percentage of patients with a confirmed partial response or better, according to the International Myeloma Working Group (IMWG) criteria. The efficacy was supported by the duration of response (DoR). The results at the primary analysis cut-off date (June 21, 2019) were the following: $31 \%$ (97.5\% CI 20.8-42.6) in the $2.5 \mathrm{mg} / \mathrm{kg}$ cohort and $34 \%$ (97.5\% CI 23.9-46.0) in the $3.4 \mathrm{mg} / \mathrm{kg}$ cohort achieved an overall response. The median follow-up was 6.3 months at the time of data cut-off, and the median DoR was not reached. $73 \%$ of responders had a DoR $\geq 6$ months. Due to a key safety concern of ocular toxicity, a Risk Evaluation and Mitigation Strategy (REMS) with Elements to Assure Safe Use (ETASU) and Communication Plan (CP) was put in place. Post-marketing requirements (PMR) were also issued for this application; some of these included the submission of the final study report and datasets from a randomized Phase 3 trial to verify the clinical benefit under accelerated approval (Sub Part E regulations) and evaluations of characterization of ocular toxicity [27].

\section{Conclusion}

As observed through the four case studies, the RTOR process is individualized to each application and is reflective of the program's pilot status. Although RTOR does not guarantee an action date prior to the PDUFA date, three out of the four cases described were approved months earlier than the PDUFA date $(2.8,3.0$, and 4.1 months for Piqray, Qinlock, and Tukysa, respectively). An advisory committee meeting was held for one of the four approved program applications, Blenrep, which was approved on the PDUFA date [27]. Thus, Blenrep was the only application that did 
not receive a time savings benefit for approval, likely due to the extended time that was required for review from the advisory committee meeting.

An additional advantage from this pilot program is the opportunity for increased communication and collaboration between the applicant and the FDA. For applications such as Piqray, there were standing biweekly teleconferences with the option for additional standing teleconferences to discuss CMC components of the application. The standing teleconferences allowed for another avenue for the Sponsor to communicate with the Agency on key topics.

These programs often participated in other pilots (AAid, Project Orbis) and other established expedited pathways during their review process. Participation in these other expedited pathways may have contributed to the time savings observed during review. In addition, interactions with the FDA and responses to information requests during the piecemeal RTOR submission process were not publicly available and were not counted in the total review time. Definitive conclusions regarding the advantages of RTOR cannot be drawn due to the small number of full NDA/BLA applications that underwent the pilot program.

While it may serve as an additional opportunity for collaboration between the Agency and Sponsor, the pilot program may increase the applicant and Agency resource burden due to the multiple interactions with the FDA prior to submission. The FDA may theoretically begin reviewing the submission components and issuing information requests ahead of the final complete application submission, which may need to be taken into consideration for resource planning. There are also certain criteria regarding the clinical trial design and development program that need to be met for the application to be reviewed under RTOR.

The publicly available data regarding RTOR was limited for these programs, especially for the programs that agreed to the RTOR process outside of the pre-NDA or pre-BLA meeting. The modules agreed with the FDA for submission were not publicly available for two of the four applications reviewed. Further research is needed to understand the impact of RTOR on applications as the number of approved applications that have participated in this pilot program is still relatively small.

Since 2018, RTOR has expanded from initially assessing only supplemental applications to new molecular entity applications in oncology. Future plans for RTOR include expanding the program to other therapeutic areas as part of the next PDUFA cycle. There are ongoing discussions with the FDA around a potential PDUFA VII follow-up program called "Split Real-Time Application Review" or STAR. According to a recent PDUFA VII Pre-Market Subgroup meeting (December 16, 2020), this program would encompass additional product types and review disciplines outside oncology and would allow for split submission and review of required sections of marketing applications [31, 32]. Certain catalysts that could potentially drive this expansion include the FDA's technology modernization program, the expansion of cloud-based submission capabilities, and the ongoing COVID-19 pandemic [33]. As demonstrated by the creation of RTOR and ongoing discussions within the Agency, new and innovative initiatives that can expedite the review and approval of medicines for patients are and will continue to be an ongoing priority for the FDA.

\section{Acknowledgements}

The authors acknowledge Concetta Freund, Kristina Vishnevetskaya, and Shivam K. Patel for their continued support.

\section{Author Contribution}

All of the listed authors contributed equally to the development of this analysis.

\section{Funding}

No funding was necessary to conduct this retrospective review.

\section{Declarations}

\section{Conflict of interest}

There was no identified financial conflict of interest among the authors of this retrospective review.

\section{Supplementary Information}

The online version contains supplementary material available at https:// doi.org/10.1007/s43441-021-00296-7.

\section{References}

1. US Food and Drug Administration (FDA). Oncology Center of Excellence. 2020. https://www.fda.gov/about-fda/fda-organizati on/oncology-center-excellence. Accessed 7 December 2020.

2. de Claro RA, Gao JJ, Kim T, et al. U.S. Food and Drug Administration: Initial experience with the Real-Time Oncology Review Program. Clinical Cancer Research. 2020. https://clincancerres. aacrjournals.org/content/clincanres/early/2020/12/02/1078-0432. CCR-20-2220.full.pdf. Accessed 7 December 2020.

3. US Food and Drug Administration (FDA). Real-Time Oncology Review Pilot Program. 2020. https://www.fda.gov/about-fda/oncol ogy-center-excellence/real-time-oncology-review-pilot-program. Accessed 7 December 2020.

4. Friends of Cancer Research. Real-Time Oncology Review and the Assessment Aid: Increase Review Efficiency Through Standardization and Earlier Data Access. 2018. https://www.focr.org/sites/ default/files/pdf/ROTR\%20White\%20Paper\%201.pdf. Accessed 7 December 2020. 
5. US Food and Drug Administration (FDA). Hematology/Oncology (Cancer) Approvals \& Safety Notifications. 2020. https://www. fda.gov/drugs/resources-information-approved-drugs/hemat ologyoncology-cancer-approvals-safety-notifications. Accessed 7 December 2020.

6. US Food and Drug Administration (FDA). Center for Drug Evaluation and Research: Piqray Multi-discipline Review. 2020. https:// www.accessdata.fda.gov/drugsatfda_docs/nda/2019/212526Orig 1s000MultidisciplineR.pdf. Accessed 7 December 2020.

7. US Food and Drug Administration (FDA). Piqray NDA Approval Letter. 2019. https://www.accessdata.fda.gov/drugsatfda_docs/ nda/2019/212526Orig1s000Approv.pdf. Accessed 7 December 2020.

8. Piqray (alpelisib). Prescribing Information. Novartis Pharmaceuticals; 2020. https://www.accessdata.fda.gov/drugsatfda_docs/ label/2020/212526s001lbl.pdf. Accessed 7 December 2020.

9. US Food and Drug Administration (FDA). FDA Approves First PI3K Inhibitor for Breast Cancer. 2019. https://www.fda.gov/ news-events/press-announcements/fda-approves-first-pi3k-inhib itor-breast-cancer. Accessed 7 December 2020.

10. US Food and Drug Administration (FDA). Center for Drug Evaluation and Research: Piqray Administrative and Correspondence Documents. 2019. https://www.accessdata.fda.gov/drugsatfda docs/nda/2019/212526Orig1s000AdminCorres.pdf

11. NIH U.S. National Library of Medicine. Study Assessing the Efficacy and Safety of Alpelisib Plus Fulvestrant in Men and Postmenopausal Women With Advanced Breast Cancer Which Progressed on or After Aromatase Inhibitor Treatment (SOLAR1). 2020 Available at: https://www.clinicaltrials.gov/ct2/show/ NCT02437318?term $=02437318 \&$ draw $=2$.

12. André F, Ciruelos E, Rubovszky G, et al. SOLAR-1 Study Group. Alpelisib for PIK3CA-Mutated, Hormone Receptor-Positive Advanced Breast Cancer. N Engl J Med. 2019;380(20):1929_ 1940. https://www.nejm.org/doi/10.1056/NEJMoa1813904. Accessed 7 December 2020.

13. US Food and Drug Administration (FDA). Center for Drug Evaluation and Research - Drug Approval Package: Tukysa. 2020. https://www.accessdata.fda.gov/drugsatfda_docs/nda/2020/21341 1Orig1s000TOC.cfm. Accessed 29 March 2021.

14. US Food and Drug Administration (FDA). FDA Approves First New Drug Under International Collaboration, A Treatment Option for Patients with HER2-Positive Metastatic Breast Cancer. 2020. https://www.fda.gov/news-events/press-announcements/fda-appro ves-first-new-drug-under-international-collaboration-treatmentoption-patients-her2. Accessed 7 December 2020.

15. Tukysa (tucatinib). Prescribing Information. Seattle Genetics; 2020. https://www.accessdata.fda.gov/drugsatfda_docs/label/ 2020/213411s000lbl.pdf. Accessed 7 December 2020.

16. US Food and Drug Administration (FDA). Tukysa NDA Approval Letter. 2020. https://www.accessdata.fda.gov/drugsatfda_docs/ appletter/2020/213411Orig1s0001tr.pdf. Accessed 7 December 2020.

17. NIH U.S. National Library of Medicine. A Study of Tucatinib vs. Placebo in Combination With Capecitabine \& Trastuzumab in Patients With Advanced HER2+ Breast Cancer (HER2CLIMB). 2020 Available at: https://clinicaltrials.gov/ct2/show/study/ NCT02614794.

18. Murthy RK, Loi S, Okines A, et al. Tucatinib, Trastuzumab, and Capecitabine for HER2-Positive Metastatic Breast Cancer. N Engl J Med 2020;382:597-609. https://www.nejm.org/doi/10.1056/ NEJMoa1914609. Accessed 7 December 2020.

19. Qinlock (ripretinib). Prescribing Information. Deciphera Pharmaceuticals; 2020. https://www.accessdata.fda.gov/drugsatfda_docs/ label/2020/213973s000lbl.pdf. Accessed 7 December 2020.

20. US Food and Drug Administration (FDA). Qinlock NDA Approval Letter. https://www.accessdata.fda.gov/drugsatfda_docs/appletter/ 2020/213973Orig1s0001tr.pdf. Accessed 7 December 2020.
21. US Food and Drug Administration (FDA). Center for Drug Evaluation and Research - Drug Approval Package: Qinlock. 2020. https://www.accessdata.fda.gov/drugsatfda_docs/nda/2020/21397 3Orig1s000TOC.cfm. Accessed 29 March 2021.

22. US Food and Drug Administration (FDA). FDA Approves First Drug for Fourth-Line Treatment of Advanced Gastrointestinal Stromal Tumors. https://www.fda.gov/news-events/press-annou ncements/fda-approves-first-drug-fourth-line-treatment-advancedgastrointestinal-stromal-tumors\#: :text=Today $\% 2 \mathrm{C} \% 20$ the $\% 20 \mathrm{U}$. S. $\% 20$ Food $\% 20$ and,originates $\% 20$ in $\% 20$ the $\% 20$ gastrointestinal $\%$ 20tract. Accessed 7 December 2020.

23. US Food and Drug Administration (FDA). Center for Drug Evaluation and Research: Qinlock Administrative and Correspondence Documents. https://www.accessdata.fda.gov/drugsatfda docs/nda/2020/213973Orig1s000AdminCorres.pdf. Accessed 7 December 2020.

24. Blay JY, Serrano C, Heinrich MC, et al. Ripretinib in patients with advanced gastrointestinal stromal tumours (INVICTUS): a double-blind, randomised, placebo-controlled, phase 3 trial. Lancet Oncol. 2020;21(7):923-934. https://doi.org/10.1016/S14702045(20)30168-6.

25. Blenrep (belantamab mafodotin-blmf). Prescribing Information. GlaxoSmithKline; 2020. https://www.accessdata.fda.gov/drugs atfda_docs/label/2020/761158s000lbl.pdf. Accessed 4 December 2020.

26. US Food and Drug Administration (FDA). FDA granted accelerated approval to belantamab mafodotin-blmf for multiple myeloma. 2020. https://www.fda.gov/drugs/drug-approvals-anddatabases/fda-granted-accelerated-approval-belantamab-mafod otin-blmf-multiple-myeloma. Accessed 4 December 2020.

27. US Food and Drug Administration (FDA). Blenrep BLA Approval Letter. 2020. https://www.accessdata.fda.gov/drugsatfda_docs/ appletter/2020/7611580rig1s0001tr.pdf. Accessed 4 December 2020.

28. US Food and Drug Administration (FDA). Center for Drug Evaluation and Research: Multi-discipline Review. 2020. https://www. accessdata.fda.gov/drugsatfda_docs/nda/2020/761158Orig1s000 MultidisciplineR.pdf. Accessed 4 December 2020.

29. US Food and Drug Administration (FDA). Center for Drug Evaluation and Research: Other Review(s). 2020. https://www.acces sdata.fda.gov/drugsatfda_docs/nda/2020/761158Orig1s000OtherR.pdf. Accessed 4 December 2020.

30. NIH U.S. National Library of Medicine. A Study to Investigate the Efficacy and Safety of Two Doses of GSK2857916 in Participants With Multiple Myeloma Who Have Failed Prior Treatment With an Anti-CD38 Antibody. 2020 Available at: https://clinicaltrials. gov/ct2/show/results/NCT03525678.

31. Gingery D. PDUFA VII Could Expand US Real-Time Review Beyond Oncology. Pink Sheet - Informa Pharma Intelligence. 2021. https://pink.pharmaintelligence.informa.com/PS143828/ PDUFAd-VII-Could-Expand-US-Real-Time-Review-BeyondOncology. Accessed 26 March 2021.

32. Prescription Drug User Fee Act (PDUFA) Reauthorization. FDA and Industry Pre-market subgroup: Meeting Summary. 2020. https://www.fda.gov/media/146127/download. Accessed 26 March 2021.

33. Sutter S. Taking the 'O' Out of RTOR: US FDA's Real-Time Review Primed For Expansion Beyond Oncology. Pink Sheet Informa Pharma Intelligence. 2020. https://pink.pharmaintellige nce.informa.com/PS142989/Taking-The-O-Out-Of-RTOR-USFDAs-Real-Time-Review-Primed-For-Expansion-Beyond-Oncol ogy?vid=Pharma\&processId $=57 \mathrm{c} 8920 \mathrm{c}-6 \mathrm{de} 6-4 \mathrm{a} 5 \mathrm{e}-80 \mathrm{ee}-\mathrm{b} 3 \mathrm{de} 5$ laabda3. Accessed 26 March 2021. 